\title{
Preparation of Gold-Tellurium Hybrid Nanomaterials for Surface-Enhanced Raman Spectroscopy
}

\author{
Zong-Hong Lin $^{\dagger}$ and Huan-Tsung Chang* $, \dagger, \ddagger$ \\ Department of Chemistry, National Taiwan University, Taipei, Taiwan, and Department of Natural Science \\ Education, National Taitung University, Taitung, Taiwan
}

Received August 16, 2007. In Final Form: November 3, 2007

\begin{abstract}
We report a simple method for preparing three different SERS-active substrates. Concentrated hydrazine solution as the reducing agent and tellurium dioxide as the precursor were used to prepare Te nanowires (NWs). The as-prepared Te NWs have an average length of $547.7 \pm 111.6 \mathrm{~nm}$ and an average width of $15.1 \pm 2.7 \mathrm{~nm}$. Through the reaction of Te NWs with sodium tetrachloroaurate in the presence of hexadecyltrimethylammonium bromide (CTAB) over reaction times of 10,20, and $60 \mathrm{~min}$, gold-tellurium nanodumbbells, gold-tellurium nanopeapods, and gold pearlnecklace nanomaterials (Au PNNs) were obtained, respectively. By controlling the reaction time, the distance between adjacent gold nanoparticles (Au NPs) in each Te nanowire was tunable, allowing us to investigate its effect on the SERS signals. Having shorter distances among Au NPs (greater electromagnetic fields), the Au PNNs provided a reproducible enhancement factor of $5.6 \times 10^{9}$.
\end{abstract}

Surface-enhanced Raman scattering (SERS) spectroscopy is a powerful analytical tool and is used when molecules are adsorbed on rough metal surfaces or on aggregated metal nanoparticles. ${ }^{1}$ Enhancements in SERS of up to $10^{14}$ are theoretically possible and have been demonstrated in some cases by applying sophisticated techniques. , $^{2,3}$ Not surprisingly, attention has turned toward the development of SERS for use as an ultrasensitive platform for the detection of biological molecules in particular. ${ }^{4}$ Among the various SERS-active metal substrates, gold and silver are used most widely. Relative to silver nanoparticles, gold nanoparticles (Au NPs) have the beneficial properties of longterm stability and a narrow size distribution. ${ }^{5}$ Nevertheless, the Raman enhancement factors of molecules adsorbed onto Au NPs are usually 100-1000 times lower than those adsorbed onto Ag NPs. To take advantage of Au and Ag nanomaterials for SERS applications, $\mathrm{Ag} / \mathrm{Au} \mathrm{NPs}^{6}$ and nanowires ${ }^{7}$ have been previously reported. However, the reproducibility and enhancement factors of SERS signals using such core/shell nanostructures are challenged.

To prepare new SERS-active metal substrates that provide strong, reproducible SERS signals of analytes, we used Te nanowires as templates and $\mathrm{Au}^{3+}$ ions as the oxidant on the basis of the fact that $\mathrm{Au}^{3+}$ ions are capable of oxidizing Te atoms in Te nanorods to form $\mathrm{TeO}_{3}{ }^{2-} .8$ In this study, we prepared three

* Corresponding author. E-mail: changht@ntu.edu.tw.Tel and Fax:011886-2-33661171.

National Taiwan University.

$\doteqdot$ National Taitung University.

(1) Moskovits, M. Rev. Mod. Phys. 1985, 57, 783

(2) Aravind, P. K.; Nitzan, A.; Metiu, H. Surf. Sci. 1981, 110, 189

(3) (a) Nie, S.; Emery, S. R. Science 1997, 275, 1102. (b) Kneipp, K.; Wang, Y.; Kneipp, H.; Perelman, L. T.; Itzkan, I.; Dasari, R. R.; Feld, M. S. Phys. Rev. Lett. 1997, 78, 1667.

(4) (a) Tuma, R. J. Raman Spectrosc. 2005, 36, 307. (b) Faulds, K.; Smith, W. E.; Graham, D. Analyst 2005, 130, 1125. (c) Baker, G. A.; Moore, D. S. Anal. Bioanal. Chem. 2005, 382, 1751. (d) Bell, S. E. J.; Sirimuthu, N. M. S. J. Am Chem. Soc. 2006, 128, 15580. (e) Faulds, K.; McKenzie, F.; Smith, W. E.; Graham, D. Angew. Chem., Int. Ed. 2007, 46, 1829.

(5) (a) Sutherland, W. S.; Winefordner, J. D. J. Colloid Interface Sci. 1992, 148, 129. (b) Mirkin, C. A.; Letsinger, R. L.; Mucic, R. C.; Storhoff, J. J. Nature 1996, 382, 607. (c) Mann, S.; Shenton, W.; Li, M.; Connolly, S.; Fitzmaurice, D. Adv. Mater. 2000, 12, 147.

(6) (a) Cui, Y.; Ren, B.; Yao, J.-L.; Gu, R.-L.; Tian, Z.-Q. J. Phys. Chem. B 2006, 110, 4002. (b) Kumar, G. V. P.; Shruthi, S.; Vibha, B.; Reddy, B. A. A.; Kundu, T. K.; Narayana, C. J. Phys. Chem. C 2007, 111, 4388.

(7) Hunyadi, S. E.; Murphy, C. J. J. Mater. Chem. 2006, 16, 3929.

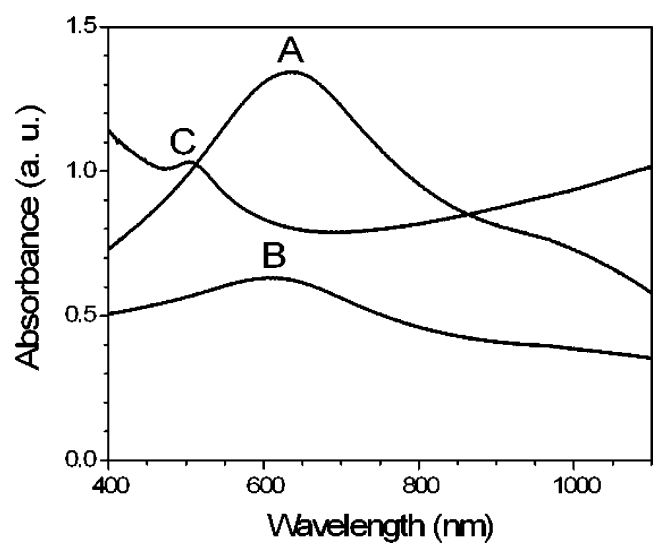

Figure 1. UV - vis absorption spectra of the synthesized (A) AuTe NDBs, (B) Au-Te NPPs, and (C) Au PNNs.

SERS-active substrates, namely, gold-tellurium nanodumbbells ( $\mathrm{Au}$-Te NDBs), gold-tellurium nanopeapods ( $\mathrm{Au}-\mathrm{Te} \mathrm{NPPs}$ ), and gold pearl-necklace nanomaterials (Au PNNs). These three nanomaterials were obtained through the reaction of Te NWs with sodium tetrachloroaurate in the presence of hexadecyltrimethylammonium bromide (CTAB) over reaction times of 10 , 20, and $60 \mathrm{~min}$, respectively. Te NWs were prepared using concentrated hydrazine solution as the reducing agent and tellurium dioxide as the precursor. The as-prepared Te NWs (Figure S1) have an average length of $547.7 \pm 111.6 \mathrm{~nm}$ and an average width of $15.1 \pm 2.7 \mathrm{~nm}$. We point out that CTAB is one of the most commonly used surfactants for growing 1D gold nanostructures. ${ }^{9}$

The UV-vis absorption spectra (Figure 1) of the three asprepared nanomaterials exhibited increased surface plasma resonance (SPR) absorbance at $505 \mathrm{~nm}$ upon increasing the amount of $\mathrm{Au}$ in the nanomaterials. The characteristic absorbance at $650 \mathrm{~nm}$ of the Te NWs ${ }^{10}$ gradually decreased and eventually

(8) Wang, Y.; Tang, Z.; Podsiadlo, P.; Elkasabi, Y.; Lahann, J.; Kotov, N. A Adv. Mater. 2006, 18, 518

(9) (a) Yu, Y.-Y.; Chang, S.-S.; Lee, C.-L.; Wang, C. R. C. J. Phys. Chem $B$ 1997, 101, 6661. (b) Mohamed, M. B.; Ismail, K. Z.; Link, S.; El-Sayed, M A. J. Phys. Chem. B 1998, 102, 9370. (c) Jana, N. R.; Gearheart, L.; Murphy, C. J. J. Phys. Chem. B 2001, 105, 4065. (d) Kim, F.; Song, J. H.; Yang, P. J. Am. Chem. Soc. 2002, 124, 14316. (e) Huang, C.-C.; Yang, Z.; Chang, H.-T. Langmuir 2004, 20, 6089. 

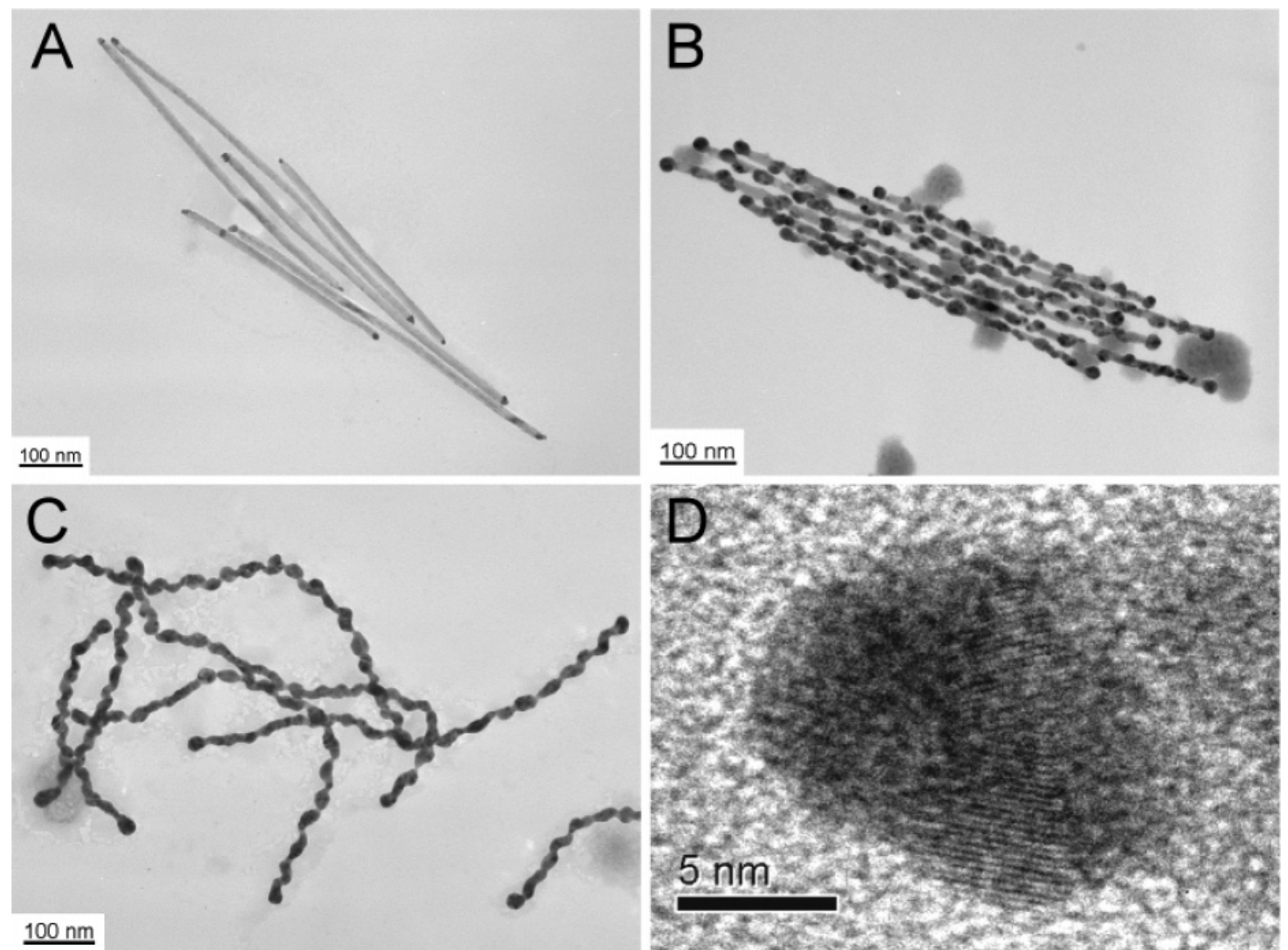

Figure 2. TEM images of SERS substrates (A) Au-Te NDBs, (B) Au-Te NPPs, and (C) Au PNNs obtained using the developed solution procedure. (D) HRTEM image of the Au NPs on Au-Te NDBs. The observed lattice spacings from the Au NPs match the spacings of the $\mathrm{Au}(111)$ planes.

disappeared upon progressing from the as-prepared Te NWs to the $\mathrm{Au}-\mathrm{Te}$ NDBs, $\mathrm{Au}-\mathrm{Te}$ NPPs, and Au PNNs. In addition, their maximum absorption wavelengths underwent blue shifts. These features resulted mainly from the increased $\mathrm{Au}$ content and decreased Te content. The absorption profile of Au PNNs is similar to that of the longer gold nanorods, which also have progressively increasing longitudinal SPR absorption in the nearIR region. ${ }^{11}$

Figure 2A indicates that spherical Au NPs having an average diameter of $12.8 \pm 2.4 \mathrm{~nm}$ grew at the ends of each Te NW through the redox reaction: $\mathrm{Au}^{3+}$ ions were reduced to $\mathrm{Au}$ atoms, and Te atoms were oxidized to form $\mathrm{TeO}_{3}{ }^{2-}$ ions. ${ }^{8}$ The similar sizes of the Au NPs suggest that the redox reaction occurs at approximately the same time on each tip. To the best of our knowledge, the prepared metal-semiconductor NDBs using our developed method are longer than the previously reported ones, ${ }^{12}$ which are shorter than $100 \mathrm{~nm}$. Besides, all of the previous approaches were demonstrated in the organic phase at high temperature. On the basis of the principle of "green chemistry", preparation at ambient temperature is favorable. Upon increasing the reaction time, more Au NPs formed on the Te NW templates.

(10) (a) Gautam, U. K.; Rao, C. N. R. J. Mater. Chem. 2004, 14, 2530. (b) Qian, H.-S.; Yu, S.-H.; Gong, J.-Y.; Luo, L.-B.; Fei, L.-F. Langmuir 2006, 22, 3830 .

(11) Wu, H.-Y.; Huang, W.-L.; Huang, M. H. Cryst. Growth Des. 2007, 7, 831.

(12) (a) Mokari, T.; Rothenberg, E.; Popov, I.; Costi, R.; Banin, U. Science 2004, 304, 1787. (b) Pacholski, C.; Kornowski, A.; Weller, H. Angew. Chem., Int. Ed. 2004, 43, 4774. (c) Mokari, T.; Sztrum, C. G.; Salant, A.; Rabani, E. Banin, U. Nat. Mater. 2005, 4, 855. (d) Carbone, L.; Kudera, S.; Giannini, C.; Ciccarella, G.; Cingolani, R.; Cozzoli, P. D.; Manna, L. J. Mater. Chem. 2006 16, 3952. (e) Saunders, A. E.; Popov, I.; Banin, U. J. Phys. Chem. B 2006, 110, 25421.
Au NPs grow onto the defect sites of the nanowire surface. Such peapod nanostructures may serve as wavelength-dependent optical nanoswitches for the SPR frequency of the encapsulated metal nanoparticles. ${ }^{13}$ The lengths of the as-prepared Au-Te NDBs, $\mathrm{Au}-\mathrm{Te}$ NPPs, and Au PNNs were $565 \pm 119,567 \pm 121$, and $574 \pm 127 \mathrm{~nm}$, respectively. Figure 2D presents an HRTEM image of the Au NPs on $\mathrm{Au}-\mathrm{Te}$ NDBs. The observed lattice spacings from the Au NPs match the expected spacings for Au(111) planes. From ICP-MS data, we confirmed the components of the three as-prepared nanomaterials. The Au mole fractions in the as-prepared $\mathrm{Au}-\mathrm{Te} \mathrm{NDBs}, \mathrm{Au}-\mathrm{Te}$ NPPs, and Au PNNs were $0.1,0.6$, and 1.0 , respectively.

We used a near-infrared (NIR) laser $(785 \mathrm{~nm})$ to obtain Raman spectra of rhodamine $6 \mathrm{G}(\mathrm{R} 6 \mathrm{G})$ on the as-prepared nanomaterials. In this study, the SERS intensities were compared under the identical number of Te nanowires. Unlike typical SERS experiments using visible lasers, recording SERS spectra in the NIR region minimizes problems associated with photochemical reactions, interference from native fluorescence, and degradation, especially for large biological molecules. ${ }^{14}$ When using the $\mathrm{Au}-$ Te NDBs as the substrate, the SERS spectrum of R6G in Figure 3 A represents an enhancement factor of only 48 , when comparing the peak area at $1364 \mathrm{~cm}^{-1}$, over that obtained through

(13) (a) Hache, F.; Ricard, D.; Flytzanis, C. J. Opt. Soc. Am. B 1986, 3, 1647 (b) Haglund, R. F.; Yang, J. L.; Magruder, R. H., III; Wittig, J. E.; Becker, K. Zuhr, R. A. Opt. Lett. 1993, 18, 373. (c) Hu, M.-S.; Chen, H.-L.; Shen, C.-H.; Hong, L.-S.; Huang, B.-R.; Chen, K.-H.; Chen, L.-C. Nat. Mater. 2006, 5, 102

(14) (a) Mahmood, U.; Weissleder, R. Mol. Cancer Ther. 2003, 2, 489. (b) Connor, E. E.; Mwamuka, J.; Gole, A.; Murphy, C. J.; Wyatt, M. D. Small 2005, 1, 325. (c) Shukla, R.; Bansal, V.; Chaudhary, M.; Basu, A.; Bhonde, R. R.; Sastry, M. Langmuir 2005, 21, 10644. (d) Qian, X.-M.; Ansari, D.; Nie, S. Proc. SPIE 2006, 6448, 644800 


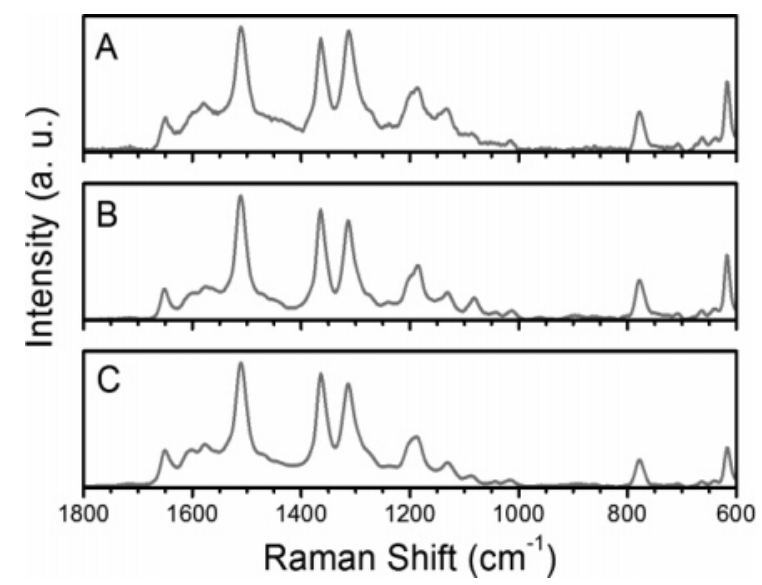

Figure 3. SERS spectra of R6G molecules adsorbed onto (A) $\mathrm{Au}-$ Te NDBs, (B) Au-Te NPPs, and (C) Au PNNs. The concentrations of R6G were $8.5 \times 10^{-6}, 9.5 \times 10^{-10}$, and $1.8 \times 10^{-11} \mathrm{M}$, respectively.

conventional Raman spectroscopy. The small enhancement effect was due to (a) the small content of Au NPs (present only at the ends of the NWs) in the as-prepared Au-Te NDBs and (b) the distance between the pair of Au NPs within each NW being too large to generate a sufficiently high electromagnetic field. Nevertheless, this type of structure might be useful in tip-enhanced Raman spectroscopy (TERS), ${ }^{15}$ which is a promising tool for nanoscale chemical analysis. The distances between pairs of adjacent Au NPs in each Au-Te NPP were shorter (average distance, ca. $23.0 \mathrm{~nm}$ ) than those in the as-prepared $\mathrm{Au}-\mathrm{Te}$ NDBs. On the basis of the knowledge that extremely intense local electromagnetic fields can be generated if the distance between adjacent metallic nanostructures (hot spots) is short, ${ }^{16}$ we expected the $\mathrm{Au}-\mathrm{Te}$ NPPs to provide more-intense SERS signals than the $\mathrm{Au}-\mathrm{Te}$ NDBs. Indeed, the SERS spectrum in Figure 3B represents a SERS enhancement factor of $2.2 \times 10^{6}$. This value is similar to that reported from SERS analyses employing Au nanodisk arrays that had been prepared using relatively complex on-wire lithography techniques. ${ }^{17}$ The SERS enhancements of our $\mathrm{Au}-\mathrm{Te}$ NPPs are, however, lower than

(15) (a) Kalkbrenner, T.; Ramstein, M.; Mlynek, J.; Sandoghdar, V.J. Microsc. 2001, 202, 72. (b) Watanabe, H.; Hayazawa, N.; Inouye, Y.; Kawata, S. J. Phys. Chem. B 2005, 109, 5012. (c) Becker, M.; Sivakov, V.; Andrä, G.; Geiger, R.; Schreiber, J.; Hoffmann, S.; Michler, J.; Milenin, A. P.; Werner, P.; Christiansen, S. H. Nano Lett. 2007, 7, 75. (d) Zhang, W.; Yeo, B. S.; Schmid, T.; Zenobi, R. J. Phys. Chem. C 2007, 111, 1733.

(16) (a) Gunnarsson, L.; Rindzevicius, T.; Prikulis, J.; Kasemo, B.; Kall, M. Zou, S. L.; Schatz, G. C. J. Phys. Chem. B 2005, 109, 1079. (b) Schuck, P. J.; Fromm, D. P.; Sundaramurthy, A.; Kino, G. S.; Moerner, W. E. Phys. Rev. Lett. 2005, 94, 017402. those of aggregated Au NPs. ${ }^{18}$ The spectrum in Figure $3 \mathrm{C}$ indicates that the largest SERS enhancement factor $\left(5.1 \times 10^{9}\right)$ resulted from the use of $\mathrm{Au}$ PNNs, in which the Au NPs were closely connected within the NWs. The SERS enhancement factor is much higher than that $\left(1.9 \times 10^{6}\right)$ using aggregates of $13 \mathrm{~nm} \mathrm{Au}$ NPs and that $\left(2.4 \times 10^{6}\right)$ using flower-shaped Ag NPs. ${ }^{19}$ It has been suggested that extremely high SERS enhancements can be achieved when interparticle distances are in the sub-10-nm range. ${ }^{20}$ Unlike the situation when using aggregated nanomaterials, the SERS signals of R6G from four different batches of $\mathrm{Au}$ PNNs (Figure S2) were reproducible (relative standard deviation, $9.6 \%$ from five runs). Under normal conditions, our limit of detection for R6G was about $2.5 \mathrm{pM}$. We predict that the detection limit would be lower if the SERS measurements were conducted under optimal conditions (i.e., using an argon ion laser with emission at $514 \mathrm{~nm}$, although such an apparatus was unavailable to us).

In summary, we report a simple method for preparing three different SERS-active substrates. By controlling the reaction time, the distance between adjacent Au NPs in each NW was tunable, allowing us to investigate its effect on the SERS signals. With shorter distances, greater electromagnetic fields were generated, which therefore led us to achieve extremely large SERS enhancements (e.g., the Au PNNs, with their multiple hot spots, provided a reproducible enhancement factor of $\left.5.6 \times 10^{9}\right)$. When using the Au PNNS, it was quite easy to find hot sites for most of the enhanced SERS signals. Typically, we required less than 2 min to obtain reproducible SERS spectra. We also highlight the fact that the preparation of these Au PNNs was simple and reproducible. With their extremely high sensitivity, reproducibility, and simplicity, it is our belief that the as-prepared $\mathrm{Au}$ PNNs hold great potential for use in bioanalysis.

Acknowledgment. This study was supported by the National Science Council of Taiwan under contracts NSC 95-2113-M002026-MY3, 96-2627-M-002-013, and 96-2627-M-002-014.

Supporting Information Available: Experimental details and TEM images of the Te NWs and different batches of Au PNNs. This material is available free of charge via the Internet at http://pubs.acs.org.

\section{LA702543K}

(17) Qin, L.; Zou, S.; Xue, C.; Atkinson, A.; Schatz, G. C.; Mirkin, C. A. Proc. Natl. Acad. Sci. U.S.A. 2006, 103, 13300.

(18) (a) Suzuki, M.; Niidome, Y.; Kuwahara, Y.; Terasaki, N.; Inoue, K.; Yamada, S. J. Phys. Chem. B 2004, 108, 11660. (b) Schwartzberg, A. M.; Grant, C. D.; Wolcott, A.; Talley, C. E.; Huser, T. R.; Bogomolni, R.; Zhang, J. Z. J. Phys. Chem. B 2004, 108, 19191.

(19) Yang, Z.; Chiu, T.-C.; Chang, H.-T. Open Nanosci. J. 2007, 1, 5.

(20) Wang, H.; Levin, C. S.; Halas, N. J. J. Am. Chem. Soc. 2005, 127, 14992. 